\title{
CBA/J Mouse
}

National Cancer Institute

\section{Source}

National Cancer Institute. CBA/JMouse. NCI Thesaurus. Code C37399.

Developed by Strong (1920), the CBA/J mouse was disseminated to Andervont (1947)

and then to Jackson Laboratory (1948). The CBA/J strain carries the gene for retinal degeneration ( $r d$ ) and has a high gross tumor incidence, including lymphoma, hepatoma, and mammary tumors. The CBA/J mice exhibit an intermediate life span with good breeding performance. 